Pesq. Vet. Bras. 30(11):988-990, novembro 2010

\title{
Ectoparasitos de animais silvestres no Maranhão ${ }^{1}$
}

\author{
Mayra A.P. Figueiredo ${ }^{2}$, Ana Clara G. Santos ${ }^{3}$ e Rita de Maria S.N.C. Guerra ${ }^{3^{\star}}$ \\ ABSTRACT.- Figueiredo M.A., Santos A.C.G. \& Guerra R.M.S.N.C. 2010. [Ectoparasites \\ of wild animals in Maranhão.] Ectoparasitos de animais silvestres no Maranhão. Pesquisa \\ Veterinária Brasileira 30(11):988-990. Departamento de Patologia, Curso de Medicina \\ Veterinária, Universidade Estadual do Maranhão, Cidade Universitária Paulo VI, Tirirical, \\ São Luis, MA 65055-970, Brazil. E-mail: grita62@ hotmail.com \\ The objective of this study was to identify the ectoparasites of wild animals received \\ by the Center of Wild Animals of São Luís, Maranhão, Brazil. The ectoparasites identified \\ were: the lice Acidoproctus sp., Trinoton sp., Ciconiphilus sp., Austromenopon sp., \\ Quadraceps sp., Saemundssonia sp., and Trichodectes canis; the ticks Amblyomma \\ rotundatum and Rhipicephalus sanguineus; the fleas Ctenocephalides felis, and larvae of \\ Cochliomyia hominivorax. The data presented register the infestation of wild mammals, \\ reptiles and birds by ectoparasites.
}

INDEX TERMS: Wild animals, ectoparasites, Maranhão

RESUMO.- Objetivou-se identificar os ectoparasitos de animais silvestres recepcionados pelo Centro de Triagem de Animais Silvestres, São Luís, Maranhão. Os ectopararasitos identificados foram: piolhos Acidoproctus sp., Trinoton sp., Ciconiphilus sp, Austromenopon sp., Quadraceps sp., Saemundssonia sp. e Trichodectes canis; carrapato Amblyomma rotundatum e Rhipicephalus sanguineus; pulgas Ctenocephalides felis e larvas de Cochliomyia hominivorax. Os resultados apresentados documentam a infestação de mamíferos, répteis e aves silvestres por ectoparasitos.

TERMOS DE INDEXAÇÃO: Animais silvestres, ectoparasitos, Maranhão.

\section{INTRODUÇÃO}

O Brasil é um dos seis países com maior diversidade biológica do mundo, com estimativas de dois milhões de espécies e aproximadamente duzentas mil catalogadas (Lewinsohn \& Prado 2002). Gerenciar este patrimônio biológico requer o estabelecimento de estratégias, planos e programas que assegurem a utilização sustentável dos recursos naturais (Dias 2001).

\footnotetext{
${ }^{1}$ Recebido em 19 de março de 2010.

Aceito para publicação em 20 de setembro de 2010.

${ }^{2}$ Bolsista PIBIC/CNPq, Universidade Estadual do Maranhão (UEMA), Cidade Universitária Paulo VI, Caixa Postal 09, Bairro Tirirical, São Luis, MA 65055-970, Brasil.

${ }^{3}$ Departamento de Patologia, Curso de Medicina Veterinária, UEMA, São Luis, MA. *Autor para correspondência: grita62@ hotmail.com.
}

Diante dessa conjuntura tem se intensificado as pesquisas sobre a fauna silvestre no Brasil, para tentar amenizar o desequilíbrio ecológico causado pela retirada histórica desses animais do hábitat natural. Os Centros de Triagens, os zoológicos e os criadouros são locais importantes para as pesquisas sobre animais silvestres em situação de cativeiro, principalmente a pesquisa clínica e laboratorial. Possibilitam conhecer as condições que podem tornar parasitos naturais em patogênicos, quando os animais estão sob o estresse do cativeiro (Arrojo 2002), evitando o óbito dos animais por doenças com agente e tratamento conhecidos (Catão-Dias 2003).

Dessa forma, o estudo das doenças parasitárias e da interação parasito-hospedeiro de animais silvestres cativos e de vida livre é uma ferramenta importante para auxiliar nos programas de conservação e preservação, prevenindo impactos negativos sobre a biodiversidade e a saúde pública (Catão-Dias 2003). A relevância da pesquisa da fauna parasitária é reconhecida e exigida em protocolos de reintrodução (UICN 1998, FELASA 1999) e na rotina clínica de animais silvestres.

O objetivo desse trabalho foi identificar os ectoparasitos dos animais silvestres recepcionados pelo Centro de Triagem de Animais Silvestres (CETAS) do Instituto Brasileiro de Meio Ambiente e Recursos Naturais Renováveis (IBAMA) de São Luís, Maranhão.

\section{MATERIAL E MÉTODOS}

O CETAS localiza-se no Bairro da Maiobinha, São Luís, Maranhão, recebe animais apreendidos pela Polícia Florestal, 
Bombeiros e da população. Por existir apenas esse centro no estado, atualmente exercer a função de centro de manejo e de triagem dos animais silvestres. As coletas de amostras ocorreram quando da admissão dos animais no CETAS. Os animais foram inspecionados no período de agosto de 2006 a julho de 2008 para pesquisa de ectoparasitos.

Para a coleta dos ectoparasitos, os animais eram inspecionados manualmente e visualmente e os espécimes coletados acondicionados em frascos com álcool a $70^{\circ} \mathrm{GL}$ e encaminhados ao Laboratório de Parasitologia Veterinária do Curso de Medicina Veterinária da Universidade Estadual do Maranhão para triagem, processamento e identificação. Em casos suspeitos de sarna e de otite parasitária realizaram-se raspados de pele e swabs de ouvido, respectivamente, para pesquisa de ácaros. A identificação dos artrópodes foi realizada com chaves propostas por Aragão \& Fonseca (1961), Furman \& Catts (1970), Linard \& Guimarães (2000) e Price et al. (2003).

\section{RESULTADOS E DISCUSSÃO}

Os resultados encontram-se sumarizados no Quadro 1.

Quadro 1. Espécies de hospedeiros examinados, números de animais amostrados, ectoparasitos identificados em animais silvestres no período de agosto/2006 a julho/2008 no Centro de triagem de Animais Silvestres (Cetas) Maranhão, Brasil

\begin{tabular}{|c|c|c|c|}
\hline $\begin{array}{l}\text { Espécie de } \\
\text { hospedeiro } \\
\text { examinado }\end{array}$ & $\begin{array}{l}\text { № de animais } \\
\text { amostrados }\end{array}$ & $\begin{array}{l}\text { № de animais } \\
\text { positivos para } \\
\text { ectoparasitos }\end{array}$ & $\begin{array}{l}\text { Ectoparasito } \\
\text { identificado }\end{array}$ \\
\hline Boa constrictor & 07 & 06 & $\begin{array}{l}\text { Amblyomma } \\
\text { rotundatum }\end{array}$ \\
\hline Iguana iguana & 01 & 01 & $\begin{array}{l}\text { Amblyomma } \\
\text { rotundatum }\end{array}$ \\
\hline Cerdocyon thous & 03 & 02 & $\begin{array}{c}\text { Trichodectes } \\
\text { canis }\end{array}$ \\
\hline Nasua nasua & 08 & 08 & $\begin{array}{c}\text { Trichodectes } \\
\text { canis }\end{array}$ \\
\hline Nasua nasua & 08 & 04 & $\begin{array}{c}\text { Ctenocephalides } \\
\text { felis }\end{array}$ \\
\hline Galactis cuja & 02 & 02 & $\begin{array}{l}\text { Rhipicephalus } \\
\text { sanguineus }\end{array}$ \\
\hline Galactis cuja & 02 & 01 & $\begin{array}{l}\text { Cochliomyia } \\
\text { hominivorax }\end{array}$ \\
\hline $\begin{array}{l}\text { Tamandua } \\
\text { tetradactyla }\end{array}$ & 01 & 01 & Amblyomma sp. \\
\hline $\begin{array}{l}\text { Thalasseus } \\
\text { maximus }\end{array}$ & 03 & 03 & Austromenopon \\
\hline $\begin{array}{l}\text { Thalasseus } \\
\text { maximus }\end{array}$ & 03 & & Quadraceps \\
\hline $\begin{array}{l}\text { Thalasseus } \\
\text { maximus }\end{array}$ & 03 & & Saemundssonia \\
\hline $\begin{array}{l}\text { Dendrocygna } \\
\text { viduata }\end{array}$ & 24 & 24 & Acidoproctus \\
\hline $\begin{array}{l}\text { Dendrocygna } \\
\text { autumnalis }\end{array}$ & 10 & 10 & Acidoproctus \\
\hline Butorides striatus & 02 & 02 & Ciconiphiluis \\
\hline
\end{tabular}

Jibóias (Boa constrictor) e iguanas (Iguana iguana) estavam infestadas por carrapatos adultos da espécie Amblyomma rotundatum, ectoparasito já identificado em outros répteis no Maranhão (Guerra et al. 2000). A literatura cita diversos hospedeiros heterotérmicos para esse carrapato (Teixeira et al. 2003, Dantas-Torres et al. 2005).
Detectou-se parasitismo por piolhos da espécie Trichodectes canis em raposinha (Cerdocyon thous) e em quati (Nasua nasua). Resultados semelhantes aos obtidos por Dominguez (2003) na Espanha que identificou em canídeos silvestres de vida livre esta espécie de piolho.

As pulgas da espécie Ctenocephalides felis foram identificada em quati. No Brasil este pulicídeo já foi registrado em quatis por Linard \& Guimarães (2000) e Rodrigues et al. (2006), estes últimos autores assinalam também o parasitismo por Rhopalopsyllus lutzi lutzi, comentam ainda que os quatis podem manter espécies de ectoparasitos e intercambiá-los entre os ambientes rural e urbano, considerando que sua amostragem foi proveniente de um fragmento de mata na área urbana.

Em furão (Galictis cuja) identificaram-se adultos Rhipicephalus sanguineus e em tamanduá-mirim (Tamandua tetradactyla) larvas Amblyomma sp. Labruna et al. (2002) relataram o encontro de Amblyomma cajennense em nove hospedeiros, Mymercophaga tridactyla, Tamandua tetradactyla, Puma concolor, Cerdocyon thous, Tayassu tajacu, Mazana gouazoubira, Hydrochaeris hydrochaeris, Alouatta caraya e Cebus apella. Torres-Mejia \& Fuente (2006) também identificaram Amblyomma sp. em $T$. tetradactyla na Colômbia, confirmando a polixevia deste gênero.

Larvas de terceiro estádio do díptero Cochliomyia hominivorax foram identificadas em lesão cutânea de furão. Este achado comprova que os animais silvestres também estão expostos as mí́ases.

As aves são freqüentemente parasitadas por piolhos. Fato que pode ser observado no presente estudo onde se constatou em trinta-réis-real (Thalasseus maximus) o parasitismo por Austromenopon sp., Quadraceps sp. e Saemundssonia sp. Resultados semelhantes foram obtidos por González-Acuña et al. (2006), no Chile, ao identificarem espécies dos gêneros Quadraceps e Saemundssonia em aves da ordem Charadriiformes, família Laridae, mesma classe taxonômica de T. maximus. Espécies dos gêneros Austromenopon e Quadraceps são comumente registradas parasitando membros da família Laridae (González-Acuña et al. 2006).

Em irerê (Dendrocygna viduata) e marreca cabocla (Dendrocygna autumnalis) verificou-se o parasitismo por pioIhos do gênero Acidoproctus Segundo Arnold (2006) o gênero Acidoproctus é freqüentemente encontrado em aves da ordem Anseriformes (famílias Anatidae e Anseranatidae), embora Brum et al. (2005) não tenham registrado este gênero nas aves anseriformes da família Anatidae (Netta peposaca e Cygnus melancoryphus) amostradas no Rio Grande do Sul, posteriormente a espécie Acidoproctus fuligulae foi assinalada neste estado por Paulsen \& Brum (2007), indicando de estudos desta natureza devem ser contínuos objetivando verificar as associações parasitárias. Valim et al. (2005) registram por primeira vez no Brasil parasitismo em Dendrocygna bicolor por Acidoproctus rostratus.

Em socozinho (Butorides striatus) identificou-se o gênero Ciconiphilus, este gênero contem espécies de pioIhos cuja distribuição está limitada a determinados hospe- 
deiros das ordens Ciciniformes e Anseriorformes. Silva et al.(2009) registraram o encontro de Ciconiphilus pectiniventris em Cygnus atratus (Anseriformes, Anatidae) e Price \& Graham (1997) relatam que várias espécies de aves das ordens Ciconiformes e Anseriformes são hospedeiros desta espécie de piolho.O gênero Trinoton foi identificado em sabiá-laranjeira (Turdus rufiventris) e irerê, e o díptero Hippoboscidae Pseudolynchia sp. em socozinho.

Segundo Valim et al. (2005), a análise de malófagos provenientes de aves apreendidas ou cativas poderá fornecer subsídios que permitam compreender a fauna de piolhos no Brasil, especialmente considerando-se a dificuldade de amostrar animais de vida livre.

A pesquisa de microscopia de pele e os swabs de ouvidos foram negativos para todos os felinos amostrados, assim como para os macacos-pregos, sagüis dos tufos brancos e raposinhas.

Estudos desta natureza contribuem para o conhecimento da fauna parasitária de animais silvestres ao tempo em que permitem estabelecer novas áreas de ocorrência e distribuição geográfica de ectoparasitos, especialmente em locais pouco estudados como o estado do Maranhão. De igual forma oportunizam conhecer os potenciais vetores de zoonoses.

\section{REFERÊNCIAS}

Aragão H.B. \& Fonseca F. 1961. Notas de Ixodologia. VII. Lista e chave para os representantes da fauna ixodológica brasileira. Mem. Inst. Oswaldo Cruz 59:115-149.

Arnold D.C. 2006. Review of the genus Acidoproctus (Phthiraptera: Ischnocera: Philopteridae), with description of the new species. J. Kansas Entomol. Soc. 79:272-282.

Arrojo L. 2002. Parásitos de animales silvestres en cativerio en Lima, Perú. Revta Peru. Biol. 2:118-120.

Brum J.B.W., Coimbra M.A., Albano A.P. \& Paulsen R.M.M. 2005. Parasitos de animais silvestres no Rio Grande do Sul: I-piolhos de alguns anseriformes. Arqs Inst. Biológico, São Paulo, 72:261-262.

Catão-Dias J.L. 2003. Doenças e seus impactos sobre a biodiversidade. Ciência e Cultura 55:32-34.

Dantas-Torres F., Oliveira-Filho E.F., Souza B.O.F. \& Sá F.B. 2005. First record of Amblyomma rotundatum Koch, 1844 (Acari: Ixodidae) parasitizing Crotalus durissus cascavella (Wagler, 1824) (Squamata: Viperidae) in the state of Pernambuco, Brazil. Arqs Inst. Biológico, São Paulo, 72:389-390.

Dias B. 2001. Demandas governamentais para o monitoramento da diversidade biológica brasileira, p.17-28. In: Garay I. \& Dias B. (Eds), Conservação da Biodiversidade em Ecossistemas Tropicais: avanços conceituais e revisão de novas metodologias de avaliação e monitoramento. Ed. Vozes, Rio de Janeiro.
Dominguez G. 2003. Ectoparásitos de los mamíferos silvestres Del Norte de Burgos (España). Galemys 15:47-60.

FELASA 1999. Health monitoring of non-human primate colonies. Laboratory Animals 33(Suppl.1):S1-S16.

Furman D.P. \& Catts E.P. 1970. Manual of Medical Entomology. Mayfiled Publishing Company. 163p.

González-Acuña D., Fisher C., Palma R., Moreno L., Barrientos C., Muñoz L., Ardoles K. \& Cicchino A. 2006. Piojos (Phthiraptera: Insecta) de aves de la familia Laridae (Aves: Charadriiformes) en Chile. Parasitología Latinoamericana 61:188-191.

Guerra R.M.SN.C., Abreu-Silva A.L. \& Serra-Freire N.M. 2000. Amblyomma rotundatum (Acari: Ixodidae) in Kinosternon scorpioides (Chelonia: Kinosternidae) in Maranhão state, Brasil. Entomol. Vect. 7:335-338.

Labruna M.B., Paula C.D., Lima T.F. \& Sana D.A. 2002. Ticks (Acari: Ixodidae) of wild animals from the Porto-Primavera Hydroeletric Power Station area, Brazil. Mem. Inst. Oswaldo Cruz 97:11331136.

Lewinsohn T.M. \& Prado P.I. 2002. Biodiversidade Brasileira: síntese do estado atual do conhecimento. Ed. Contexto, São Paulo. $176 p$.

Linard P.M. \& Guimarães L.R. 2000. Sifonápteros do Brasil. FAPESP, São Paulo, p.291.

Paulsean R.M.M. \& Brum J.G.W. 2007. Parasitos de animais silvestres no Rio Grande do Sul, Brasil. II. Piolhos (Amblycera: Ischnocera) de Netta peposaca (marreção) (Aves: Anaetidae). Arqs Inst. Biológico, São Paulo, 74:35-37.

Price M.A. \& Graham O.H. 1997. Chewing and sucking lice as parasites of mammals and birds. Technical Bull. 1849, USDA Agricultural Research Service. 257p.

Price R.D., Hellenthal R.A., Palma R.L., Johnson K.P. \& Clayton D. H. 2003. The chewing lice: world checklist and biological overview. Natural Survey Special Publication, Illinois. 501p.

Rodrigues A.F.S.F., Daemon E. \& Massard C.L. 2006. Ectoparasites of Nasua nasua (Carnivora, Procyonidae) from an urban forest in southeastern Brazil. Arq. Bras. Med. Vet. Zootec. 58:969-971.

Silva S.O., Oliveira H.H., Teixeira R.H.F. \& Amorim M. 2009. Malófagos (Phthiraptera, Amblycera, Ischnocera) em aves cativas no sudeste do Brasil. Revta Bras. Entomol. 53:495-497.

Teixeira R.H.F., Amorim M., Gazeta G.S. \& Serra-Freire N.M. 2003. Ixodofauna de répteis cativos do Zoológico de Sorocaba, São Paulo, Brasil. Entomol. Vect. 10:319-329.

Torres-Mejia A.M. \& Fuente J. 2006. Risks associated with ectoparasites of wild mammals in the Departamento of Quindio, Colombia. Int. J. Appl. Res.Vet. Med. 4:187-192.

UICN 1998. Guias para reintroducciones de la UICN: preparada por el Grupo de especialistas en Reintroducción de la Comision de Supervivencia de Especies de la UICN. UICN, Gland, Suiza, y Cambridge, Reino Unido.

Valim M.P., Teixeira R.H.F., Amorim M. \& Serra-Freira N.M. 2005. Malófagos (Phthiraptera) recolhidos de aves silvestres no Zoológico de São Paulo, SP, Brasil. Revta Bras. Entomol. 49:584-587. 